------ Raf. J. Sci., Vol. 27, No.1, pp.73-81, 2018------

\title{
Reduction of Nonlinear Optical Self Phase Modulation in Optical Fiber at 40 Gb/s
}

\author{
Muhammed S. Hameed \\ Department of Physics / College of Science / University of Mosul
}

E-mail: mohamed.subhy1@yahoo.com

(Received 28/10 / 2013 ; Accepted 16/12 / 2013 )

\begin{abstract}
A nonlinear Schrodinger equation has been solved in order to establish the nonlinear optical self phase modulation (SPM) actual magnitude in fiber (within optical domain), the SPM magnitude was simulated at $40 \mathrm{GHz}$ in relation to normalized time and actual distance within the fiber. When the SPM signal comes out of the fiber and enters a pre-designed transimpedance amplifier (TIA) (electronic domain), and according to simulation, the SPM signal corresponds to an input noise spectral density of $320.3 \mathrm{pA}^{2} / \mathrm{Hz}$ (electronic domain) that enters the TIA. A significant reduction in $\mathrm{S}_{21}$ scattering parameter, nonlinear optical power and nonlinear noise figure were registered when the $320.3 \mathrm{pA}^{2} / \mathrm{Hz}$ is entered at the TIA input. That effectively means a reduction in SPM value using the TIA at $40 \mathrm{GHz}$. A Microwave Office software was used for simulation.
\end{abstract}

Keywords: SPM, Nonlinear Optical, NLO Impairments.

\section{تخفضض تضفمن اللور الذاتي البصري اللذالي اللف الضوئي عند 40كيكلبلت بالثانية}

\section{المالغص}

لهات معادلةشرونككر اللاخطية لغرض تحديد القيمة القققية لتضمين الطور الذلي البصري اللانطي (SPM) في اللي ف

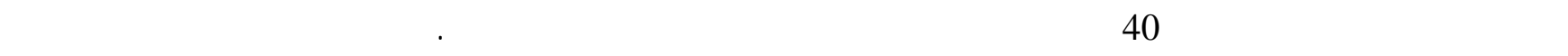

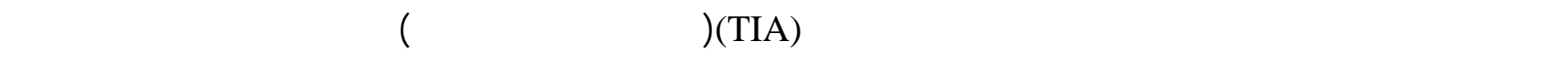

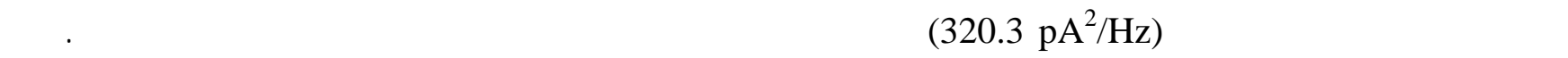
الحصول على خفض كبير في كل من معالمل النتت S21 والقدرة البصرية اللإطية وكذك النخفاض ملحوظ في قيمة الـ لـضوضاء اللاضطية عند قيمة النظ (TIA) مما يعني انخفاضاً في تضمين الطور الذاني الب صصري اللاطي SPM تم لستخدلم بررنامج (Microwave Office) في النمنجة.

الكالمات الدالة: تضضمن الطور الذالي، بصرية لاخطية، معوقلت بصرية لاظطية.

\section{INTRODUCTION}

Experimental performance evaluation of SPM compensation and its application in optical transport network was introduced by (Yamazaki et al., 2010). Experimental results showed transmission distances of $100 \mathrm{~Gb} / \mathrm{s}$ signal reaches 16.340 and $12.015 \mathrm{~km}$ in single channel 10 channel wavelength division multiplexing (WDM) conditions respectively (Yamazaki et al., 2010).

A double cell, self-phase modulation based optical regenerator was designed and optimized to reduce signal noise and distortion. Investigation on multiple passes through the proposed optical 
regenerator showed significant amplitude jitter suppression compared single pass alternatives. An improvement in Q-factor became negligible after only five loops for this system and a double Q-factor is achieved demonstrating the efficiency of the proposed scheme (Bolt and Ensser, 2010).

A multilevel coded modulation system in the presence of nonlinear phase noise for fiber optical communication was introduced. The scheme exploited a 16-point ring constellation with nonlinear post compensation of the self-phase modulation via the Kerr effect. A new set partitioning based on the Ungerboeck approach was introduced to maintain unequal error protection in amplitude and phase direction. Simulation results showed that the proposed system provides up to $2 \mathrm{~dB}$ gain over a forward error correcting scheme for a block error rates around $10^{-9}$, with the same overhead (7\%) and complexity (Beygi et al., 2010).

The effect of dispersion slope of highly nonlinear fiber on the performance of self-phase modulation based 2R-optical regenerator was investigated numerically. This study showed that the dispersion slope has a significant impact on the shape of the transfer function of the regenerator. The dispersion slope can therefore be used together with the dispersion value and the filter offset as an additional parameter to control the performance of the regenerator. (Ghafoor and Petropoulos, 2010).

An analytical study of self phase modulation (SPM) and cross-phase modulation (XPM) was performed for coherent Differential Phase Shift Keying DPSK and ON-OFF (OOK) signal in NonReturn to Zero (NRZ) and Return-to Zero (RZ) modulation format for wavelength division multiplexing (WDM) system employing distributed Raman amplifier (DRA). The study showed that 40 Gb/s RZ-DPSK signal with 33.3\% duty cycle experiences minimum XPM and SPM induced crosstalk. The results also revealed that minimum crosstalk was induced in backward pumped DRA among the three pumping schemes i.e forward, backward and bi-directional. Results assumed importance for minimizing deleterious XPM and SPM effects in optical communication system (Anamika, 2013).

\section{Self Phase Modulation in Optical Domain}

For pulse evolution in an optical fiber, the nonlinear Schrodinger (NLS) equation governs that as follows (Agrawal, 2001):

$$
i \frac{\partial U}{\partial z}=\frac{1}{2} \beta_{2} \frac{\partial^{2} U}{\partial T^{2}}-e^{-a z}|U|^{2} U
$$

$U$ is the pulse optical field within the fiber, $z$ is distance that the pulse moves in fiber, $T$ is pulse periodic time, $\beta_{2}$ is the dispersion coefficient and $\alpha$ is the attenuation coefficient. The actual optical field envelope in fiber is given as (Agrawal, 2001):

$$
U(z, T)=\frac{T_{0}}{\left[T_{0}^{2}-i \beta_{2} z(1+i c)\right]^{1 / 2}} \exp \left[-\frac{(1+i c) T^{2}}{2\left(T_{0}^{2}-i \beta_{2} z(1+i c)\right)}\right]
$$

The chirping frequency $c$ is set at $1 . T_{o}$ is considered to be the pulse width.

To start deriving the value equivalent to the self phase modulation (SPM) magnitude which is $e^{-\alpha z}|U|^{2} U$ (given in equation 1), equation 2 can be used in which the following $s_{1}$ and $s_{2}$ parameters can be identified: 


$$
\begin{aligned}
& s_{1}=\frac{T_{a}}{\left[T_{o}^{2}-i \beta_{2} z(1+i)\right]^{1 / 2}}=\frac{T_{a}}{\left(T_{o}^{2}+\beta_{2} z(1-i)\right)^{1 / 2}} \\
& s_{2}=\exp \left[-\frac{(1+i) T^{2}}{2\left[T_{0}^{2}-i \beta_{2} z(1+i)\right]}\right]=\exp \left[-\frac{(1+i) T^{2}}{2\left[T_{0}^{2}+\beta_{2} z(1-i)\right]}\right]
\end{aligned}
$$

$\frac{\partial U}{\partial z}$ equation is derived as:

$$
\frac{\partial U}{\partial z}=s_{1} \frac{\partial s_{2}}{\partial z}+s_{2} \frac{\partial s_{1}}{\partial z}
$$

To satisfy equation (3), the values of $\frac{\partial s_{z}}{\partial z}$ and $\frac{\partial s_{z}}{\partial z}$ can be obtained:

$$
\begin{aligned}
& \frac{\partial s_{1}}{\partial z}=\frac{\frac{1}{2} T_{0} \beta_{2}(1-i)}{\left(T_{a}^{2}+\beta_{2} z(1-i)\right)^{3 / 2}}=\frac{T_{0} \beta_{2}(1-i)}{2\left(T_{a}^{2}+\beta_{2} z(1-i)\right)^{3 / 2}} \\
& \frac{\partial s_{2}}{\partial z}=\frac{1(1+i) \beta_{2} T^{2}(1-i)}{2} \frac{\operatorname{loxp}\left[\frac{-(1+i) T^{2}}{\left.2\left[T_{0}^{2}+\beta_{2} z(1-i)\right]^{2} z(1-i)\right]}\right]}{=\frac{\beta_{2} T^{2}}{\left[T_{0}^{2}+\beta_{2} z(1-i)\right]^{2}} \exp \left[\frac{-(1+i) T^{2}}{2\left[T_{0}^{2}+\beta_{2} z(1-i)\right]}\right]}
\end{aligned}
$$

Therefore, from equation (3),

$$
\frac{\partial U}{\partial z}=\frac{\beta_{2} T_{0} T^{2}}{\left[T_{0}^{2}+\beta_{2} z(1-i)\right]^{3 / 2}}+\frac{\beta_{2} T_{0}(1-i)}{2\left[T_{0}^{2}+\beta_{2} z(1-i)\right]^{3 / 2}} \exp \left[\frac{-(1+i) T^{2}}{2\left[T_{0}^{2}+\beta_{2} z(1-i)\right]}\right] \text { (4) }
$$

After defining $\frac{\partial v}{\partial z}$ which is part of the left hand side of equation (1), the right hand side terms of equation (1) can be defined. Beginning with $\frac{\hat{\sigma}^{2} v}{\partial T^{2}}$, the $\frac{\partial U}{\partial T}$ value is derived first as follows: 


$$
\frac{\partial U}{\partial T}=s_{1} \frac{\partial s_{2}}{\partial T}=\frac{-T_{o} T(1+i)}{\left[T_{o}^{2}+\beta_{2} z(1-i)\right]^{3 / 2}} \exp \left[\frac{-(1+i) T^{2}}{2\left[T_{o}^{2}+\beta_{2} z(1-i)\right]}\right]
$$

Hence,

$$
\frac{\partial^{2} U}{\partial T^{2}}=\left[\frac{2 T_{0} T^{2}}{\left[T_{0}^{2}+\beta_{2} z(1-i)\right]^{5 / 2}}-\frac{T_{o}(1+i)}{\left[T_{0}^{2}+\beta_{2} z(1-i)\right]^{3 / 2}}\right] \exp \left[\frac{-(1+i) T^{2}}{2\left[T_{0}^{2}+\beta_{2} z(1-i)\right]}\right](6)
$$

The other term part of equation (1) which is $|U|^{2} U$ can be obtained as follows:

$$
\begin{aligned}
& |U|^{2}=U^{*} \cdot U=\frac{T_{o}^{2}}{\left[T_{0}^{2}+\beta_{2} z(1-i)\right]} \exp \left[\frac{-T^{2} T_{o}^{2}}{\left(T_{o}^{2}+\beta_{2} z\right)^{2}+\beta_{2}^{2} z^{2}}\right] \\
& |U|^{2} U=\frac{T_{o}^{3}}{\left[T_{o}^{2}+\beta_{2} z(1-i)\right]^{3 / 2}} \exp \left[\frac{-2 T_{o}^{2} T^{2}}{\left(T_{o}^{2}+\beta_{2} z\right)^{2}+\beta_{2}^{2} z^{2}}\right] \exp \left[\frac{-(1+i) T^{2}}{2\left[T_{0}^{2}+\beta_{2} z(1-i)\right]}\right]
\end{aligned}
$$

By multiplying $|U|^{2} U$ by $e^{-a z}$, the magnitude of SPM can be obtained. To simulate the magnitude $e^{-\alpha z}|U|^{2} U$ against $\frac{T}{T_{c}}$ and $z$, the following typical values (at $40 \mathrm{~Gb} / \mathrm{s}$ ) can be set. $\beta_{2}$ is set to 15 $\mathrm{ps}^{2} / \mathrm{km}$, the pulse width $T_{0}$ is set to $25 \mathrm{ps}$ (@40 GHz), the pulse period $T$ is considered to range from 25 ps to $100 \mathrm{ps}, \alpha=0.02$ at wavelength of $1.54 \mu \mathrm{m}$. The value of $z$ can be up to 0.4 meter. Fig. 1 illustrates the magnitude $e^{-\alpha z}|U|^{2} U$ (SPM value) against pulse time ratio $\frac{T}{T_{o}}$ and distance $z$.

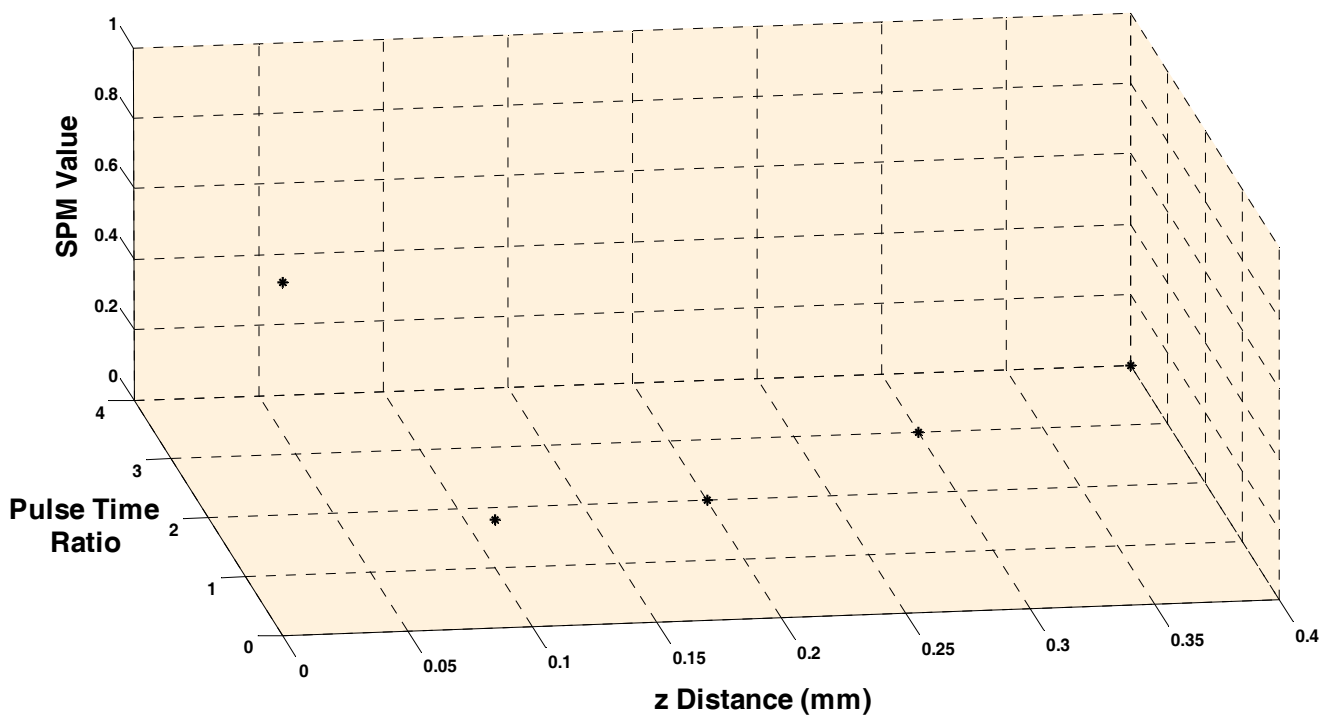

Fig. 1: Self Phase Modulation evolution in terms of pulse time ratio $\frac{T}{T_{0}}$ and distance $z$. 


\section{SPM Reduction at Electronic Domain}

When the signal comes out of the fiber (optical domain carrying SPM noise) and enters a transimpedance (TIA) amplifier (electronic domain), the following work deals with the reduction of that noise. A schematic diagram of a transimpedance (TIA) amplifier electronic circuit is shown in Fig. (2). The RLC FET transistor circuit is commonly used for several purposes, however, it has not been used before as an SPM reducing mechanism. Furthermore, the transistor type used in this work is a high-speed STATZ GaAs FET transistor (Statz et al., 1987) which is used in this work as a new application of TIA amplifier. It is useful to say that $R_{F}=148.2 \mathrm{Ohm}$ (feedback resistor value), $\mathrm{L}_{\mathrm{F}}=0.3$ $\mathrm{nH}$ (feedback inductance), $\mathrm{C}_{\mathrm{F}}=91 \mathrm{pF}$ (feedback capacitance), and $\mathrm{C}_{\mathrm{i}}=1 \mathrm{pF}$ (input capacitance).

STATZ GaAs FET transistor characteristics can be described in which under normal operating conditions the gate-to-source capacitance is much larger than the gate-to-drain capacitance. At zero drain-to-source voltage, both capacitances are about equal. For negative drain-to-source voltages, the original source acts like a drain and vice versa. Consequently, the normally large gate-to-source capacitance becomes small and acts like a gate-to-drain capacitance. In order to model these effects, it is necessary to realize that there are no separate gate-to-source and gate-to-drain charges, but that there is only one gate charge which is a function of gate-to-source and gate-to-drain voltages (Statz et al., 1987).

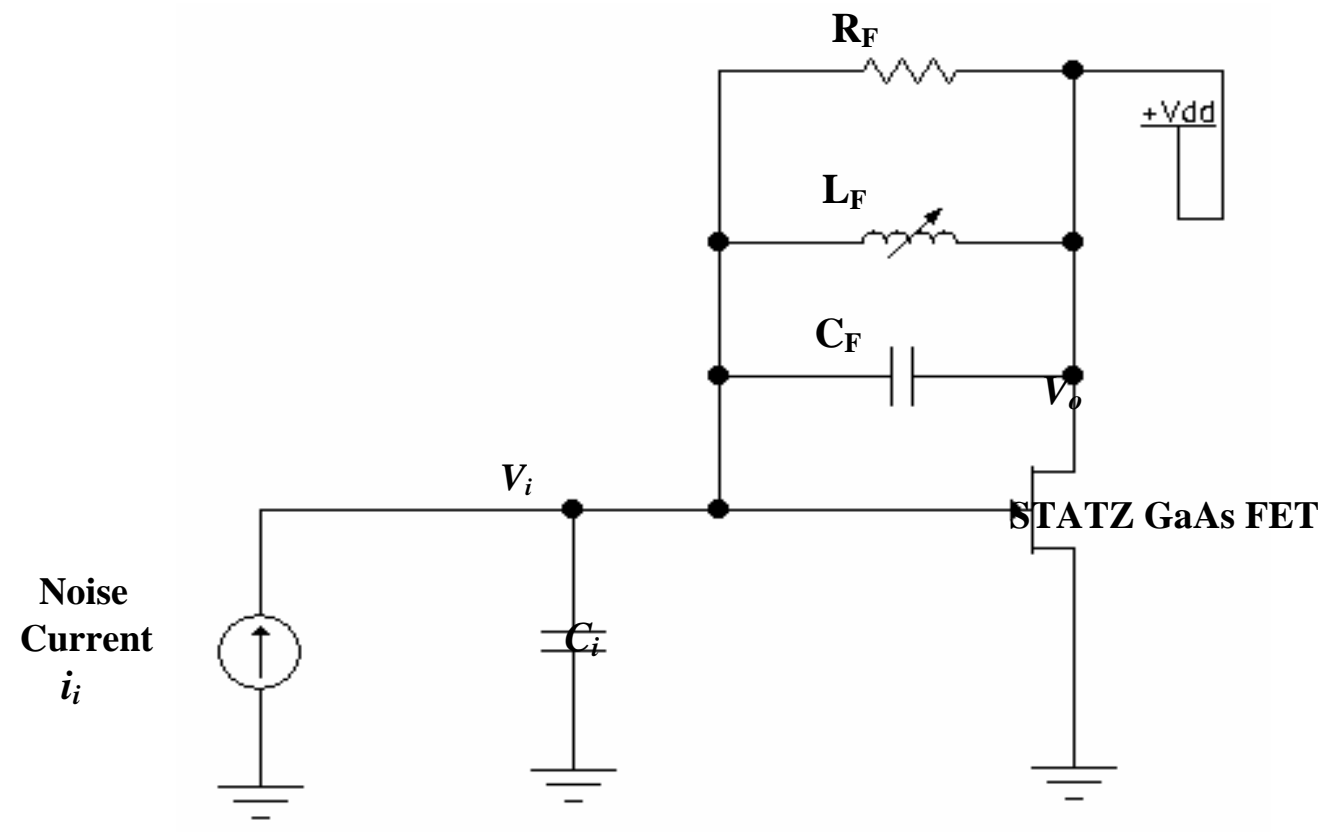

Fig. 2: Transimpedance amplifier schematic design.

In the TIA circuit, the simulated input noise current $\mathrm{i}_{\mathrm{i}}$ is entered to correspond to a value of the actual magnitude of SPM which is $e^{-a z}|U|^{2} U$ (from optical domain). The best value that worked well (in simulation within electronic domain) for an input noise spectral density was $320.3 \mathrm{pA}^{2} / \mathrm{Hz}$. We can consider that $C_{i}$ is the input capacitance, $V_{i}$ is the input noise voltage (transformed SPM). The output voltage is denoted as $V_{o}$.

The transimpedance inverting amplifier voltage gain is given as (Razavi, 2006):

$$
\frac{V_{o}}{V_{i}}=-A(s)=-A_{0} \frac{\omega_{0}}{s+\omega_{0}}
$$


For which $s=j \omega$ is the complex frequency variable, $A_{0}$ is the amplifier open loop amplifier gain, $A(s)$ is the closed loop frequency dependent amplifier gain, while the value of $\omega_{0}=2 \pi f_{0}$ in which $f_{o}$ is the open loop cut off frequency. The transfer function $\frac{v_{o}}{i_{i}}$ of the TIA circuit can be derived in order to show how much of the input noise current is transformed into output voltage (TIA gain $\frac{V_{g}}{i_{i}}$ ). From electronic circuits principles, the sum of currents at the $V_{i}$ node is:

$$
i_{i}=\frac{V_{i}}{\frac{1}{s C_{i}}}+\frac{V_{i}-V_{0}}{\frac{1}{\frac{1}{R_{F}}+\frac{1}{s L_{F}}+s C_{F}}}
$$

From equations (9) and (10), we have:

$$
i_{i}=\frac{V_{o}}{-A} s C_{i}+\left(\frac{V_{0}}{-A}-V_{0}\right)\left[\frac{s^{2} R_{F} L_{F} C_{F}+s L_{F}+R_{F}}{s R_{F} L_{F}}\right]
$$

Multiplying by $R_{F}$,

$$
\begin{gathered}
i_{i} R_{F}=\frac{V_{0}}{-A} s R_{F} C_{i}+\left(\frac{V_{0}}{-A}-V_{0}\right)\left[\frac{s^{2} R_{F} L_{F} C_{F}+s L_{F}+R_{F}}{s L_{F}}\right] \\
i_{i} R_{\vec{F}}=\frac{V_{0}}{-A} s R_{F} C_{i}-\frac{V_{0}(1+A)}{A}\left[\frac{s^{2} R_{F} L_{F} C_{F}+s L_{F}+R_{F}}{s L_{F}}\right] \\
i_{i} R_{F}=-\frac{V_{0}}{-A}\left[s R_{F} C_{i}+(1+A)\left[\frac{s^{2} R_{F} L_{F} C_{F}+s L_{F}+R_{F}}{s L_{F}}\right]\right]
\end{gathered}
$$

Obtaining $\frac{v_{0}}{i_{i} R_{F}}$ :

$$
\frac{V_{0}}{i_{i} R_{F}}=-A\left[\frac{1}{s R_{F} C_{i}+(1+A)\left[\frac{s^{2} R_{F} L_{F} C_{F}+s L_{F}+R_{F}}{s L_{F}}\right]}\right]
$$

Hence,

$$
\begin{aligned}
& \frac{V_{o}}{i_{i} R_{F}}=-A\left[\frac{1}{\left.\frac{s^{2} R_{F} L_{F} C_{i}+(1+A)\left(s^{2} R_{F} L_{F} C_{F}+s L_{F}+R_{F}\right)}{s L_{F}}\right]}\right] \\
& \frac{V_{O}}{i_{i} R_{F}}=-A\left[\frac{s L_{F}}{s^{2} R_{F} L_{F} C_{i}+(1+A)\left(s^{2} R_{F} L_{F} C_{F}+s L_{F}+R_{F}\right)}\right] \\
& \frac{V_{O}}{i_{i} R_{F}}=-A\left[\frac{s L_{F}}{s^{2} R_{F} L_{F} C_{i}+s^{2} R_{F} L_{F} C_{F}+s L_{F}+R_{F}+A\left(s^{2} R_{F} L_{F} C_{F}+s L_{F}+R_{F}\right)}\right]
\end{aligned}
$$

We replace $A$ by $A_{0} \frac{\omega_{\sigma}}{s+\omega_{o}}$ as in equation (9): 


$$
\begin{aligned}
& \frac{V_{o}}{i_{i} R_{F}}=-A_{0} \frac{\omega_{o}}{s+\omega_{o}}\left[\frac{s L_{F}}{s^{2} R_{F} L_{F} C_{i}+s^{2} R_{F} L_{F} C_{F}+s L_{F}+R_{F}+A_{0} \frac{\omega_{Q}}{s+\omega_{0}}\left(s^{2} R_{F} L_{F} C_{F}+s L_{F}+R_{F}\right)}\right] \\
& \frac{V_{o}}{i_{i} R_{F}}=\left[\frac{-s L_{F} A_{Q} \omega_{o}}{\left(s+\omega_{0}\right)\left[s^{2} R_{F} L_{F} C_{i}+s^{2} R_{F} L_{F} C_{F}+s L_{F}+R_{F}\right]+A_{0} \omega_{0}\left(s^{2} R_{F} L_{F} C_{F}+s L_{F}+R_{F}\right)}\right]
\end{aligned}
$$

Multiply by $R_{F}$

$$
\frac{V_{Q}}{i_{i}}=\left[\frac{-s R_{F} L_{F} A_{Q} \omega_{o}}{\left(s+\omega_{Q}\right)\left[s^{2} R_{F} L_{F} C_{i}+s^{2} R_{F} L_{F} C_{F}+s L_{F}+R_{F}\right]+A_{Q} \omega_{Q}\left(s^{2} R_{F} L_{F} C_{F}+s L_{F}+R_{F}\right)}\right]
$$

Further simplification indicates that the transfer function of the TIA amplifier is:

$$
\frac{V_{o}}{i_{i}}=\frac{-s R_{F} L_{F} A_{\theta} \omega_{o}}{A s^{3}+B s^{2}+C s+D}
$$

For which:

$$
\begin{aligned}
& A=\left(R_{F} L_{F} C_{i}+R_{F} L_{F} C_{F}\right) \\
& B=\left(L_{F}+\omega_{0} R_{F} L_{F} C_{i}+\omega_{0} R_{F} L_{F} C_{F}+A_{0} \omega_{\theta} R_{F} L_{F} C_{F}\right) \\
& C=\left(R_{F}+\omega_{0} L_{F}+A_{0} \omega_{0} L_{F}\right) \\
& D=\omega_{0} R_{F}\left(1+A_{0}\right)
\end{aligned}
$$

As mentioned earlier, the best value that worked well (in simulation within electronic domain) for an input noise spectral density was $320.3 \mathrm{pA}^{2} / \mathrm{Hz}$. Therefore, the value of SPM (from optical domain) $e^{-a z}|U|^{2} U$ should correspond to $320.3 \mathrm{pA}^{2} / \mathrm{Hz}$ of input noise spectral density (electronic domain) in order to have an efficient reduction in SPM. In order to study the reduction in SPM using the TIA configuration, two aspects must be taken into account. The first is how does the $S_{21}$ scattering parameter behave and secondly, what would be the nonlinear noise output power actual magnitude. The $\mathrm{S}_{21}$ noise spectrum shows the noise gain reduction for SPM corresponding to $320.3 \mathrm{pA}^{2} / \mathrm{Hz}$ of input current spectral density (electronic domain) at $40 \mathrm{GHz}$. Figure 3 illustrates the reduction of $\mathrm{S}_{21}$ noise spectrum (corresponding to SPM noise at optical domain) at $40 \mathrm{GHz}$, while at lower frequencies, $\mathrm{S}_{21}$ noise spectrum is much higher. At the same time, Fig. (3) includes the TIA gain (transfer function given in equation 14) versus frequency.

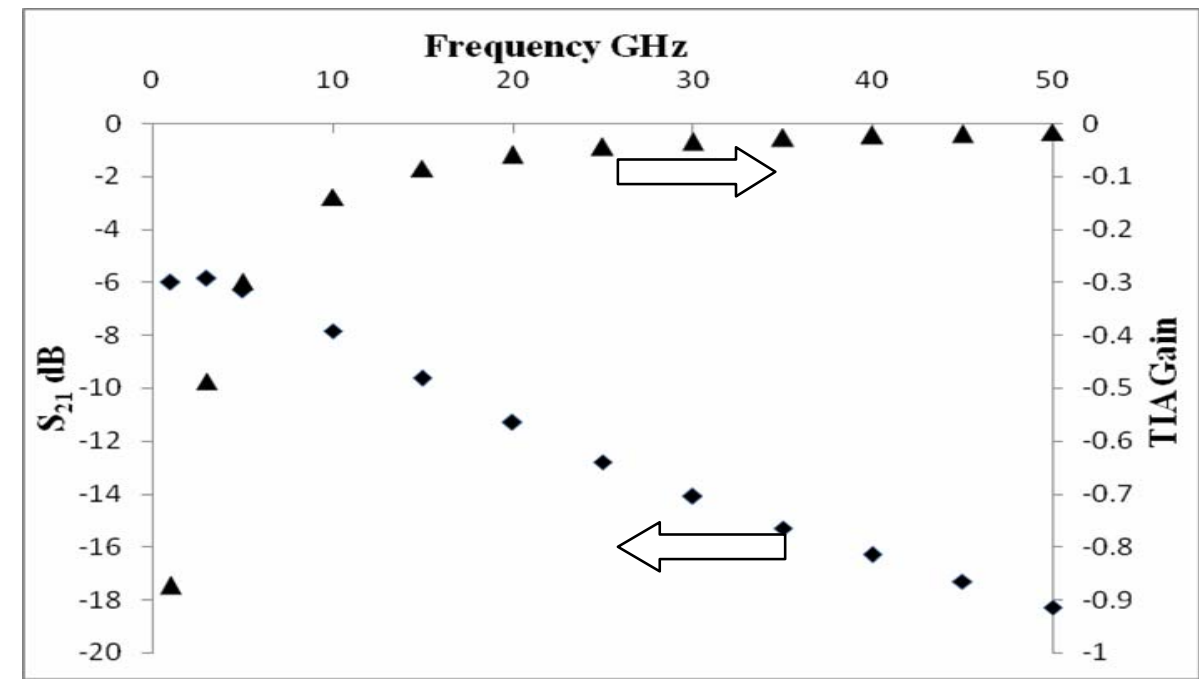

Fig. 3: $S_{21}$ noise spectrum reduction at $40 \mathrm{GHz}$ compared to lower frequencies 
From Fig. (3), several comments can be made. To start with, the negative sign of the TIA gain means that the TIA amplifier is an inverting one, and that is clear from the minus sign in equation 14. Furthermore, the TIA gain maintains its strength beyond $40 \mathrm{GHz}$ which is a good efficiency indication. It is important to notice that along side the rise in TIA gain of main signal, there is a downward direction of the noise $S_{21}$ parameter which is highly useful in terms of noise reduction capability. At 40 $\mathrm{GHz}$, both TIA gain and noise $\mathrm{S}_{21}$ scattering parameter are the subject of study in this work, although data shown at $50 \mathrm{GHz}$ have shown equally important results. Main fiber optic communication systems focus on $40 \mathrm{~Gb} / \mathrm{s}$ data bit rate and that explains the reason behind the $40 \mathrm{GHz}$ frequency point. However, the range of up to $50 \mathrm{GHz}$ covers a wide area of fiber optic high data bit rate operating conditions.

The nonlinear noise output power of the transimpedance amplifier when SPM noise is applied that corresponds to $320.3 \mathrm{pA}^{2} / \mathrm{Hz}$ (electronic domain) is shown in Figure 4. A reduction in the nonlinear noise output power is clearly shown at $40 \mathrm{GHz}$ (up to $50 \mathrm{GHz}$ ) compared to lower frequencies.

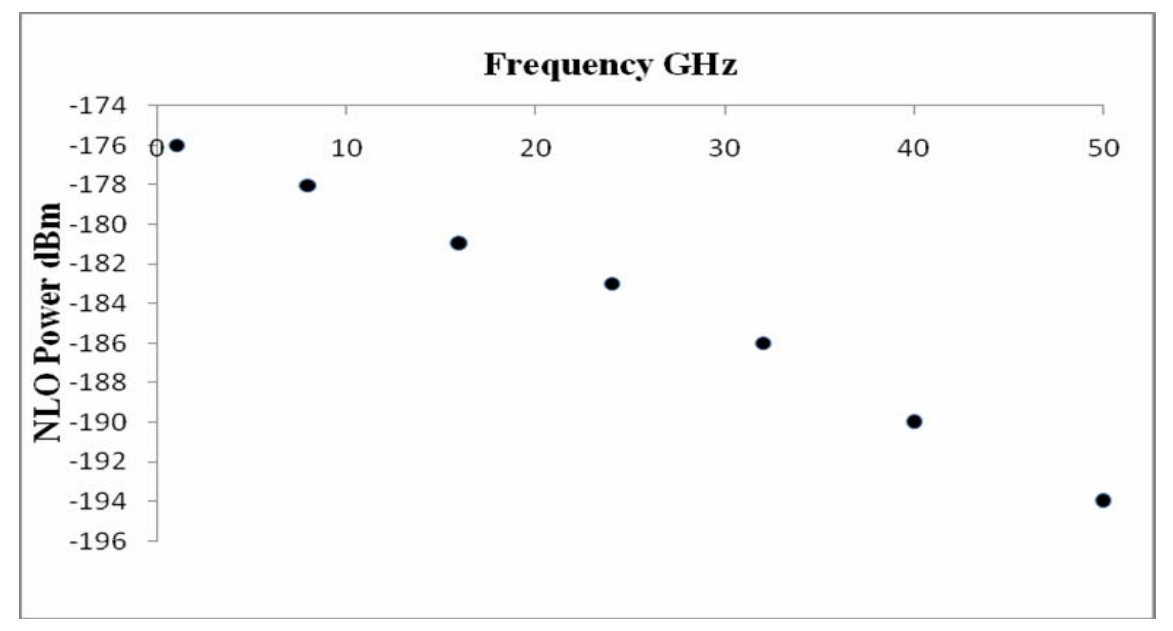

\section{Fig. 4: Nonlinear noise output (NLO) power reduction at $40 \mathrm{GHz}$ compared to lower frequencies.}

The nonlinear noise figure (NF) is reduced as in Fig. (5). That implicitly means a real reduction in SPM value, while the transfer function magnitude (equation 14 as TIA gain of main signal) is also embedded in which the TIA gain rise in an opposite direction of the reduced nonlinear noise figure (NF). There is a significant and important gap between the TIA gain and NF at $40 \mathrm{GHz}$. Further analysis of Fig. (5) indicates that it is possible to make use of data not only at $40 \mathrm{~Gb} / \mathrm{s}$ but it can also be useful to do that at $10 \mathrm{~Gb} / \mathrm{s}$. Although that analysis below $5 \mathrm{GHz}$ may not be that useful due to the fact that the gap between the TIA gain and the nonlinear noise figure (NF) gets narrow. Finally, from Figs. (3 \& 5), an inverse relationship between noise (in terms of SM) and TIA gain develops most importantly at $40 \mathrm{GHz}$ and even beyond that. The concept of noise reducing transimpedance amplifier is therefore implemented in this work. 


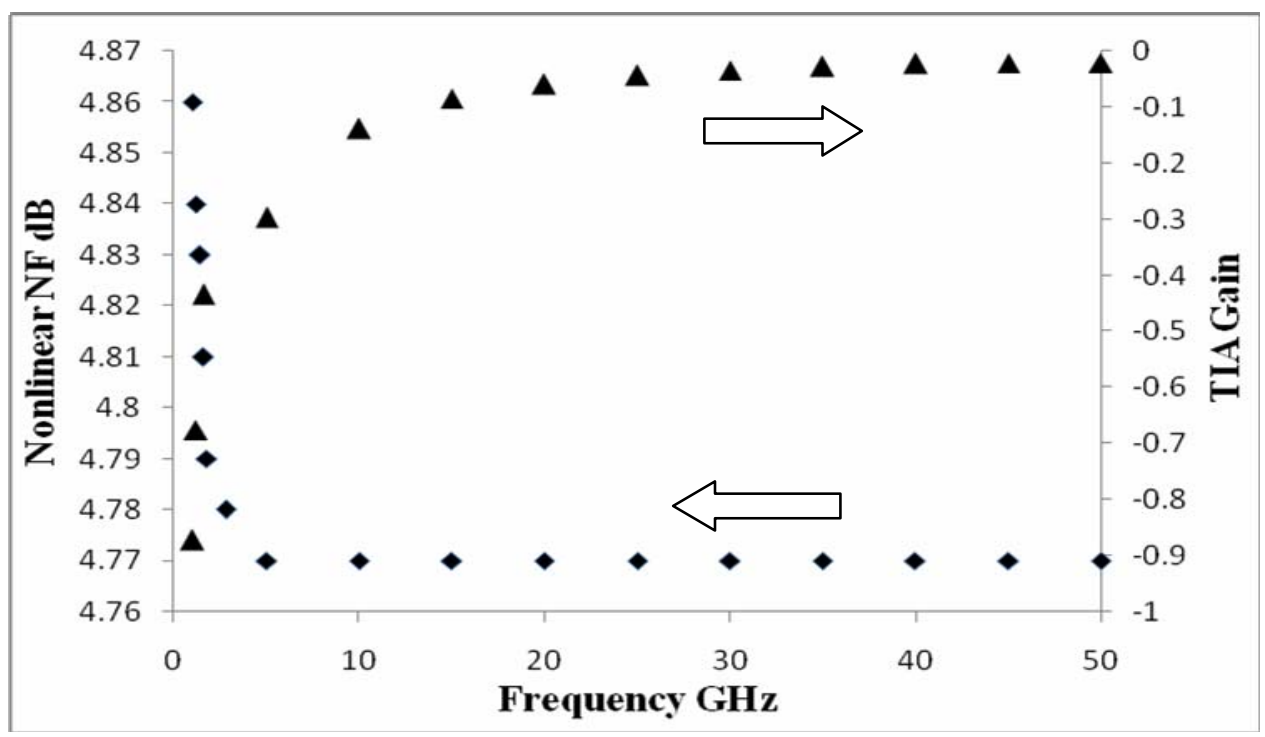

Fig. 5: Nonlinear Noise Figure (NF) reduction range up to $40 \mathrm{GHz}$.

\section{CONCLUSION}

The actual magnitude of self phase modulation (SPM) can be established for $40 \mathrm{GHz}$ signal in an optical fiber. It was confirmed that a significant reduction in SPM can occur when the signal is applied to a STATZ GaAs FET based transistor transimpedance amplifier (TIA). Furthermore, this SPM reduction was demonstrated through the actual reduction in $\mathrm{S}_{21}$ scattering parameter, nonlinear optical power and nonlinear noise figure. Another advantage of the TIA system is that alongside the noise reduction, there was a TIA gain (main signal) derived from the TIA transfer function. Although that the input noise spectral density of $320.3 \mathrm{pA}^{2} / \mathrm{Hz}$ is proved to be a good indication of the TIA system capability for noise reduction, however, future research may be possible in improving the system sensitivity in terms of input noise.

\section{REFERENCES}

Anamika, V. (2013). XPM and SPM induced crosstalk in WDM system employing distributed raman amplifier for DPSK and OOK modulation format. Optical fiber Technology, 19(2), 75-82.

Agrawal', G.P. (2001). "Nonlinear Fiber Optics". 3rd edn., Academic Press publishing, 109 p.

Bolt, D.; Ennser. K. (2010). "Investigation of Multiple Passes Through a Double-Cell, Self-Phase Modulation Based Regenerator". 12th International Conference on Transparent Optical Networks, Publisher: IEEE, pp. 1-4.

Beygi, L.; Agrell, E.; Johannisson, P.; Karlsson, M. (2010). "A Novel Multilevel Coded Modulation Scheme for Fiber Optical Channel with Nonlinear Phase Noise". 2010 IEEE Global Telecommunications Conference GLOBECOM, Publisher: IEEE, pp.1-6.

Ghafoor, S.; Petropoulos, P. (2010). "Effect of Dispersion Slope of Highly Nonlinear Fiber on the Performance of Self Phase Modulation Based 2R-Optical Regenerator". 2010 2nd International Conference on Computer Technology and Development, Publisher: IEEE, pp. 144-148.

Razavi, B. (2006). "Fundamentals of Microelectronics". Wiley Publications, 415 p.

Statz, H.; Newman, P.; Smith, I.W.; Pucel, R.A. (1987). GaAs FET device and circuit simulation in SPICE. IEEE Trans. Electron Devices, 34(2), 160-169.

Yamazaki, E.; Sano, A.; Kobayashi, T.; Miyamoto, Y. (2010). "SPM Compensation of NO-GuardInterval PDM Co-OFDM for Optical Transport Network". IEEE Photonics Society Summer Tropicals, pp. 10-11. 